\title{
Survival Analysis after Core Decompression in Association with Platelet-Rich Plasma, Mesenchymal Stem Cells, and Synthetic Bone Graft in Patients with Osteonecrosis of the Femoral Head
}

\author{
Riccardo D’Ambrosi ${ }^{1,2}$ Elena Biancardi ${ }^{1,2}$ Giulia Massari ${ }^{3}$ Vincenza Ragone ${ }^{4}$ Renato Mario Facchini ${ }^{2}$ \\ ${ }^{1}$ Dipartimento di Scienze Biomediche per la Salute, Università degli \\ Studi di Milano, Milan, Italy \\ ${ }^{2}$ Centro Traumatologico Ortopedico, U.O. Clinica Ortopedica e

\begin{abstract}
Address for correspondence Riccardo D'Ambrosi, MD, Dipartimento di Scienze Biomediche per la Salute, Università degli Studi di Milano,
\end{abstract} \\ Milan, Italy (e-mail: riccardo.dambrosi@hotmail.it).
}

Traumatologica, Milan, Italy

3 Università degli Studi di Milano, Milan, Italy

${ }^{4}$ IRCCS Policlinico San Donato, San Donato Milanese, Italy

Joints 2018;6:16-22.

\begin{abstract}
Keywords

- femoral head osteonecrosis

- core decompression

- platelet-rich plasma

- mesenchymal stem cells

- synthetic bone graft

Purpose The aim of this study was to report the rate of survivorship in patients with osteonecrosis of the femoral head treated with core decompression in association with mesenchymal stem cells (MSCs) implantation, platelet-rich plasma (PRP) injection, and synthetic bone graft.

Methods We evaluated 24 hips in 16 patients, according to Ficat classification, treated by core decompression, injection of PRP and MSCs, and backfilling of the core tract with synthetic bone graft. Survivorship was estimated using Kaplan-Meier curves. Results The survivorship of core decompression in association with the procedure is $50 \%$ at 75 months of follow-up. The survival rate was $80 \%$ for patients in early stage and $28.6 \%$ for patients in advanced stage at 75 months. When we compared Kaplan-Meier survival curves of patients in stage III + IV and patients in stage I + II, we noticed that the survival functions are statistically different ( $p<0.05$, log-rank test), particularly in stage I + II where we had a greater surviving core decompression, in comparison to patients in stage III + IV.

Conclusion This technique is safe and good preliminary results were obtained in patients with early stages of the disease with no reported complications.

Level of Evidence Level IV, therapeutic case series.
\end{abstract}

\section{Introduction}

Osteonecrosis of the femoral head (ONFH) is a disorder with a wide-ranging etiology and the pathogenesis is still unclear. ${ }^{1}$ Estimates indicate that 10,000 to 20,000 new cases are diagnosed in the United States each year. ${ }^{2}$

Glucocorticoid use and alcohol abuse are among the most recognized risk factors for this disease. Different pathophysiological mechanisms have been postulated, including fat

received

August 14, 2017

accepted after revision

January 2, 2018

published online

February 12, 2018 emboli, increase of intraosseous pressure, and microfracture of the trabecular bone. ${ }^{2}$

ONFH is commonly seen in patients between their third and sixth decades and it can lead to destruction of the hip joint, with femoral head collapse and coxarthrosis. Usually the symptom reported by these patients is pain, often localized to the groin; this pain can limit range of motion (ROM), especially passive internal rotation, greatly reducing the normal activities of daily living and therefore the quality of life.

Copyright $\odot 2018$ Georg Thieme Verlag License terms KG Stuttgart · New York

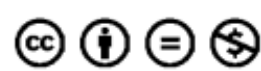


The diagnosis is performed with a radiographic evaluation in association with magnetic resonance imaging (MRI). ${ }^{1-3}$ The most common method of staging the disease is Ficat classification, which recognizes five different stages of bone necrosis from stage 0 to stage $4 .^{4}$

The gold standard for end-stage osteonecrosis is total hip arthroplasty (THA). ${ }^{5}$ Despite the clinical success of THA in this population, significant concerns persist regarding the long-term outcomes of younger patients undergoing prosthetic joint arthroplasty. ${ }^{5,6}$ These concerns lead to practice nonoperative management like physical therapy, or a conservative surgical approach, like core decompression, with the goal of delaying or preventing the need for THA. Even though this procedure has been used for more than three decades, its efficacy remains controversial and there is still no consensus in literature. ${ }^{7,8}$ One of the possible reasons for its failure is that a single core decompression does not induce an adequate osteogenic activity in the necrotic area. The development of regenerative medicine has gone beyond this limit and in recent years core decompression has been supplemented with additional procedures like an osteoinductive agent that can enhance bone repair. ${ }^{9}$ Bone healing is produced by a cellular mechanism including mesenchymal stem cells (MSCs). The MSCs need to be recruited in the pathological area. These nonhematopoietic progenitor cells are able to be differentiated in osteoblasts under the influence of growth factors such as bone morphogenetic proteins, platelet-derived growth factor (PDGF), transforming growth factor $\beta$ (TGF- $\beta$ ), insulinlike growth factor, fibroblast growth factor (FGF), and parathyroid hormone. ${ }^{9,10}$

For these reasons, we adopted a surgical approach that provides core decompression, performed with an expandable reamer tool that allows optimal debridement of dead bone through a small incision, in association with MSCs implantation and platelet-rich plasma (PRP) injection. The association of MSCs and PRP induces an osteogenic activity and stimulates bone repair. PRP is a fraction extracted by the centrifugation of whole blood, which contains a high concentration of platelets; three to four times higher than normal. The growth factors present in platelets, such as PDGF, TGF- $\beta$, basic FGF (bFGF), endothelial growth factor (EGF), and vascular EGF (VEGF), play a critical role in tissue repair, regeneration, and differentiation of MSCs. ${ }^{9-13}$

The procedure is completed with a synthetic bone graft (PRO-DENSE bone graft substitute; Wright Medical Group, Memphis, Tennessee, United States) for backfilling the surgically created defect. PRO-DENSE is a new $\mathrm{CaSO}_{4} / \mathrm{CaPO}_{4} \mathrm{com}$ posite graft that provides a bone graft substitute that resorbs and is replaced with bone during the healing process.

The aim of this study was to report the survivorship in patients with ONFH treated with core decompression in association with MSCs implantation, PRP injection, and synthetic bone graft substitute and to define the role of this procedure in avoiding or delaying THA.

Our hypothesis was that a new CaSO4/CaPO4 synthetic composite graft, in association with PRP and autologous mesenchymal cells during core decompression, could delay total hip replacement in patients with $\mathrm{OFNH}$, especially in patients with early stages of the disease.

\section{Methods}

We prospectively evaluated the results of a series of 16 patients with ONFH, treated by core decompression, injection of PRP and MSCs, and backfilling of the core tract with PRO-DENSE.

The institutional review board approved the study protocol and all patients gave informed consent for participation in the study.

The indication for the procedure was ONFH of all stages according to Ficat classification. ${ }^{4}$

Exclusion criteria included femoral head with advanced segmental collapse; patients with post-traumatic ONFH; patients aged 65 years or more; presence of blood dyscrasias or a history of vascular disease; chemotherapy anticancer therapies in progress or drugs that inhibit bone marrow function; sepsis, septic arthritis, osteomyelitis, or other ongoing infectious processes; other systemic infectious processes; previous hip operations, both in open or arthroscopy; women who were pregnant; patients deemed mentally incapable and/or for which it was reasonably foreseeable that they would not adhere to the planned program of postoperative evaluation; preoperative platelet count $<175,000 / \mu \mathrm{L}$ and hemoglobin $<11 \mathrm{~g} / \mathrm{dL}$.

There were 14 men ( 22 hips) and 2 women ( 4 hips). The mean age at the time of the procedure described was 42 years (range 29-60 years; standard deviation \pm 9.8 ). Right hips were involved in 14 cases (53.8\%), while left hips in the remaining 12 (46.2\%). We identified the following risk factors and associated conditions with ONFH: three patients were on chronic therapy with steroids (defined as a dose $>2 \mathrm{~g}$ prednisone or its equivalent per month for 3 months minimum; underlying pathologies were rheumatoid arthritis and Steinert dystrophy); two patients were HIV positive and three were alcohol abusers. In the remaining eight patients, the necrosis was idiopathic.

The diagnosis of ONFH was made using anteroposterior radiographs and standard MRI. The hip stage was classified according to Ficat classification. ${ }^{4}$ We had two cases $(7.7 \%)$ of stage I, eight cases $(30.8 \%)$ of stage II, ten cases $(38.5 \%)$ of stage III, and six cases (23.1\%) of stage IV (-Table $\mathbf{1}$ ).

Among the patients with bilateral osteonecrosis, two had both hips in stage II, two had a hip in stage II and stage III in the contralateral, and the other six had a hip stage III and the contralateral stage IV. The average time elapsed between the first surgery and surgery on the contralateral hip was 12.2 months, with a minimum of 2 months and a maximum of 18 months. The interval minimum of 2 months was in the two patients suffering from necrosis in stage III and IV.

\section{Surgical Technique}

The first step of the surgical procedure was taking a $60 \mathrm{cc}$ venous blood sample; after centrifugation, using the GPS III Platelet Separation System (Biomet Biologics, Warsaw, Indiana, United States) a formulation of PRP was obtained with over $90 \%$ of the available platelets. 
Table 1 Demographic and epidemiologic data

\begin{tabular}{|c|c|}
\hline \multicolumn{2}{|l|}{ Sex } \\
\hline Male & $14(87.5 \%)$ \\
\hline Female & $2(12.5 \%)$ \\
\hline \multicolumn{2}{|l|}{ Etiology } \\
\hline Idiopathic & $8(50 \%)$ \\
\hline Chronic therapy with steroids & $3(18.7 \%)$ \\
\hline HIV positive & $2(12.6 \%)$ \\
\hline Alcohol abusers & $3(18.7 \%)$ \\
\hline \multicolumn{2}{|l|}{ Side } \\
\hline Right & $14(53.8 \%)$ \\
\hline Left & $12(46.2 \%)$ \\
\hline Age at surgery $(y)$ & $41.9 \pm 9.8$ \\
\hline \multicolumn{2}{|l|}{ Ficat classification } \\
\hline 1 & $2(7.7 \%)$ \\
\hline II & $8(30.8 \%)$ \\
\hline III & $10(38.4 \%)$ \\
\hline IV & $6(23.1 \%)$ \\
\hline Mean follow-up (y) & $4.2 \pm 1.8$ \\
\hline
\end{tabular}

Abbreviation: HIV, human immunodeficiency virus.

With the patient under lumbar or general anesthesia, placed on the operating table in a supine position on a radiolucent table, in a sterile surgical field, aspiration of $60 \mathrm{cc}$ of autologous bone marrow in the anterior superior iliac crest was performed. The next step was isolation and concentration of aspirated marrow to obtain MSCs. After centrifugation, red blood cells and plasma are removed to keep only the nucleated cells, which are stem cells, monocytes, and lymphocytes. The concentrate of the mononucleated component thus obtained has a volume of approximately $6 \mathrm{~mL}$. The resulting transplant material has a concentration of mononuclear cells 6.9 times greater than the concentration present in the aspirated bone marrow starting.

The procedure was performed during the same surgery time of core decompression of the femoral head. With the patient placed on the operating table, a $3 \mathrm{~cm}$ longitudinal incision was made at the level of the greater trochanter and after the subcutaneous diaeresis, the fascia lata and the vastus lateralis were dissected until the lateral cortex of the proximal femur was reached. Under fluoroscopic guidance (both anteroposterior and lateral views), a Kirschner wire was introduced, as a guide wire into the necrotic lesion.

At this point, after the introduction of the tissue protector over the guide wire, the decompression of the necrotic area was performed with a $9 \mathrm{~mm}$ drill bit, monitoring the direction under fluoroscopic control. The tip of the trephine was stopped at a distance of $5 \mathrm{~mm}$ from the endosteal surface of the femoral head.

Afterward, the necrotic zone was thoroughly cleared using a sharp curette and the X-REAM (Wright Medical
Group) percutaneous expandable reamer for advanced debridement. Fluoroscopic guidance was very useful at this stage, helping to estimate how thoroughly the necrotic zone had been cleared. Once debridement was complete, the suction tip from the PRO-DENSE Core Decompression Procedure Kit was used to remove the debrided tissue, and flushing with a combination of irrigation and suction was performed. At this moment, pre-prepared PRP and MSCs were injected into the core. Then the bone core, soaked in MSCs, was turned, and synthetic (PRO-DENSE) injectable graft was used to completely fill the surgically created

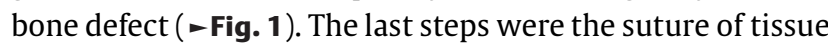
layers and the wound medication.

\section{Postoperative Rehabilitation}

Patients were given a physiotherapy protocol to strengthen the gluteal muscles and quadriceps after surgery and began to walk with aids without loading on the side operated on, by on average of 40 days. For the following 3 months, progressive tolerance loading was recommended. The indications for the load were the same for all patients, regardless of the stage of the disease at diagnosis.

\section{Statistical Analysis}

Longitudinal analysis using Kaplan-Meier estimates and a log-rank test was used to compare survival curves of patients with different Ficat stages, where survival rate refers to the intervention of total hip replacement. The association between the ONFH stage and the failure rate of core decompression treatment in association with PRP and MSCs injection and synthetic bone graft substitute was investigated by Fisher's exact test. For data analysis, a dedicated statistical software was used (SPSS vs 19, Chicago, Illinois, United States).

\section{Results}

Two hips of grade IV, in patients with bilateral disease, were excluded from the study because they underwent directly to prosthetic surgery. Twenty-four hips were enrolled in the study.

The rate of failure was statistically significantly higher in patients with disease at stage III and IV compared with patients at stage I and II $(p<0.05)$. The survivorship of core decompression in association with the procedure is $50 \%$ at 75 months of follow-up (-Fig. 2). The survival rate was $80 \%$ for patients in early stage and $28.6 \%$ for patients in advanced stage at 75 months. From the 24 hips included in the study, in 12 cases (50\%) joint replacement was necessary: two patients in stage II (25\%) were scheduled for total hip replacement after 2.5 years from core decompression, six patients were in stage III(60\%), of which four patients underwent hip replacement after 2.4 years and two patients scheduled for surgical procedure after 6 years from core decompression, and four patients in stage IV underwent hip replacement after 1.4 years (100\%). Overall patients undergo THA at a mean of 2.7 years (-Table 2 ). When we compared Kaplan-Meier survival curves of patients in stage III + IV and patients in stage I + II, we noticed that the survival 


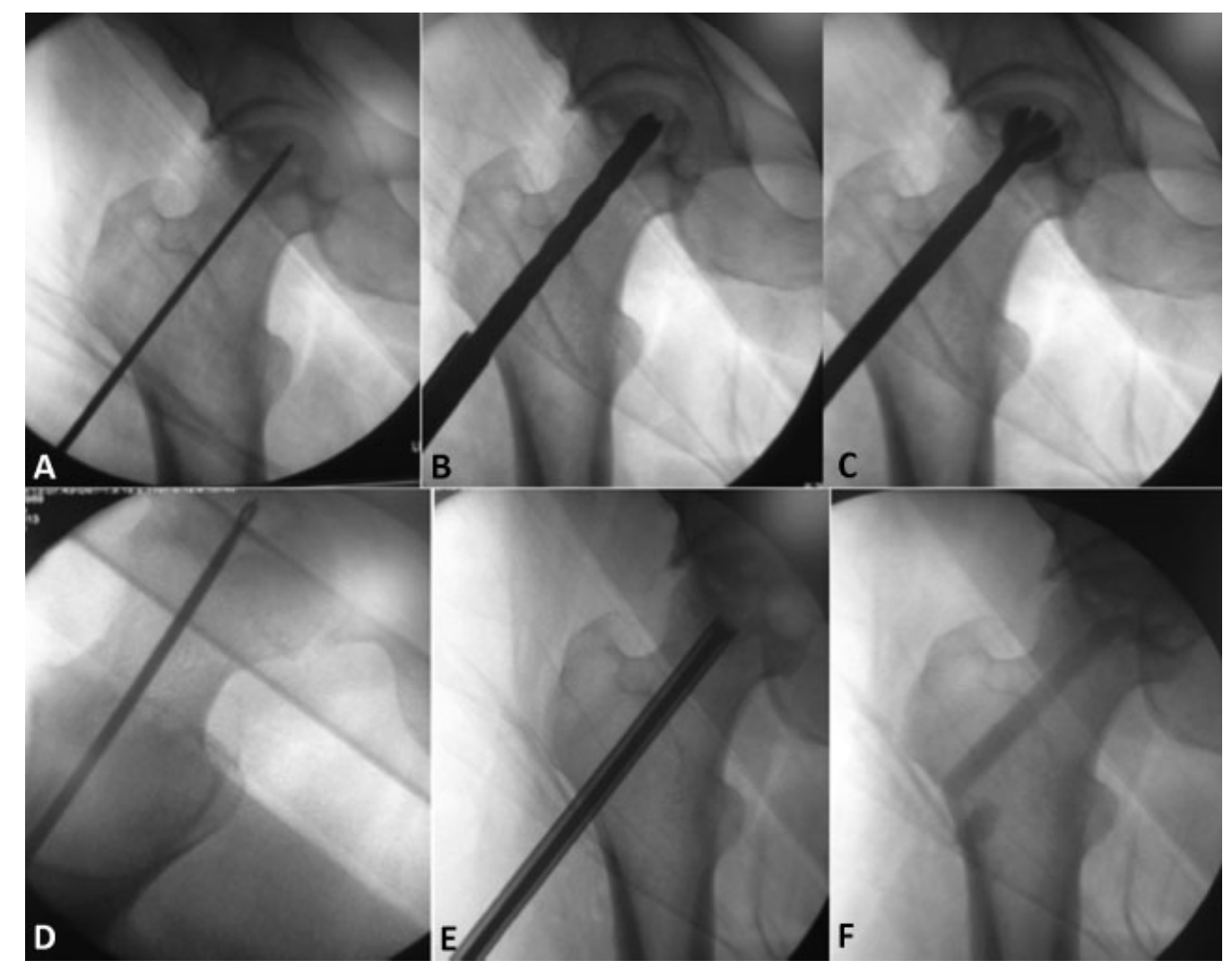

Fig. 1 Surgical main step of the procedure: (A) Introduction of a Kirschner wire as a guide wire into the necrotic lesion; (B) decompression of the necrotic area with a $9 \mathrm{~mm}$ drill bit and (C) cleaning using a sharp curette and the X-REAM percutaneous expandable reamer for advanced debridement; (D) suction tip used to remove the debrided tissue and flushing with a combination of irrigation and suction; (E) injection of PRP and MSCs, in the core; (F) postoperative radiographic control.

functions are statistically different $(p<0.05, \log$ rank test), particularly in stage I + II where we had a greater surviving core decompression, in comparison to patients in stage III + IV (-Fig. 3).

\section{Discussion}

This study investigates the effectiveness of core decompression treatment in association with mesenchymal cell implan-

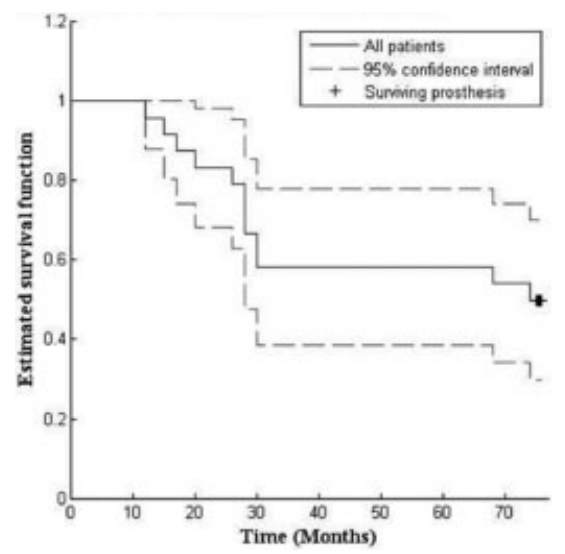

Fig. 2 Kaplan-Meier survival curves for all patients at 75 months for prosthesis, after core decompression. tation, PRP injection and synthetic bone graft substitute in avoiding or delaying THA in patients with ONFH.

It has been demonstrated that core decompression, managed in the early stage of osteonecrosis, reduces the intraosseous pressure in the femoral head and promotes vascular invasion and regeneration of the necrotic tissue. ${ }^{14-16}$ Mont evaluated 42 studies in which a total of 1,206 hips were treated with core decompression and 819 had various nonoperative treatments. ${ }^{2}$ Seventy four percent of the hips treated prior to collapse resolved adequately after core decompression, and only $22.2 \%$ of the hips treated nonoperatively had satisfactory outcomes.

Table 2 Failure rate in 24 operated hips

\begin{tabular}{|l|l|}
\hline Stage & Failure $\boldsymbol{n}$ (\%) \\
\hline I + II $(n=10$ hips) & $2(20)$ \\
\hline III + IV ( $n=14$ hips $)$ & $10(71.4)^{\mathrm{a}}$ \\
\hline I ( $n=2$ hips $)$ & $0(0)$ \\
\hline II $(n=8$ hips $)$ & $2(25)$ \\
\hline III ( $n=10$ hips $)$ & $6(60)^{\mathrm{b}}$ \\
\hline IV ( $n=4$ hips $)$ & $4(100)$ \\
\hline
\end{tabular}

Note: Failure has been defined as if arthroplasty was performed after core decompression, or the patient was scheduled for hip replacement. ${ }^{a} p<0.05$ stage I + II versus stage III + IV.

${ }^{\mathrm{b}} p>0.05$ stage III versus stage I + II. 


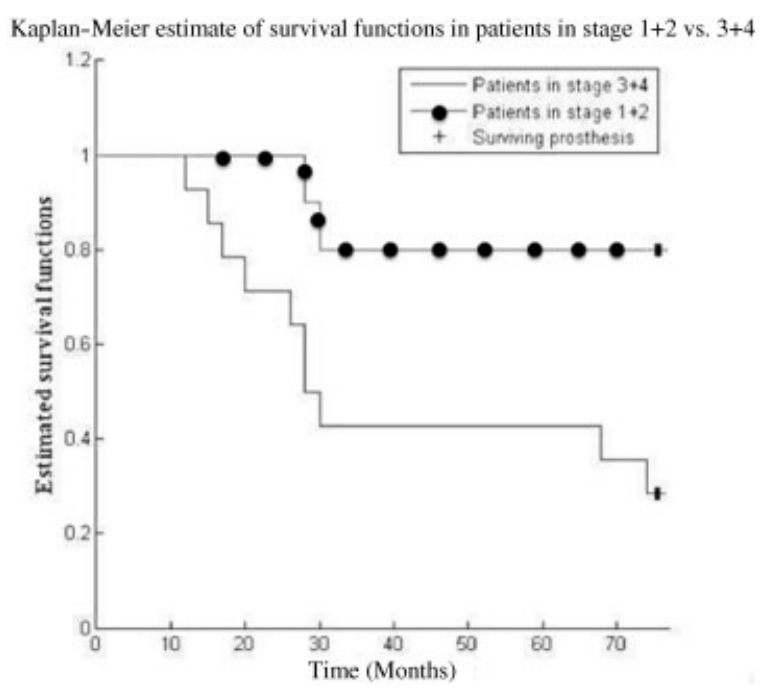

Fig. 3 Comparison of survival curves at 75 months for core decompression.

The core decompression alone does not induce an adequate osteogenic activity in the necrotic area and it has been proven that subchondral mechanical support in the weight-bearing segment of the femoral head is essential during the period of revascularization to prevent femoral head collapse. ${ }^{17}$ A metaanalysis that evaluated core decompression alone found that further surgical intervention was necessary in 16, 37, and 71\% of Steinberg stages I, II, and III, respectively. ${ }^{18}$

In 2010, Bednarek evaluated 63 patients ( 72 hips) with aseptic osteonecrosis of the femoral, and all patients were treated with core decompression, followed by filling the bone defect with autologous or synthetic bone grafts (PRO-DENSE). ${ }^{19}$ After 1 year of follow-up, pain relief with preservation of a spherical femoral head was obtained in 45 hips (63\%). Recently Yu evaluated 19 hips in 18 patients with ONFH, 6 hips in stage II C and 13 hips in stage III A, graded according to the system of the Association Research Circulation Osseous and estimated by the modified index of necrosis, treated with core decompression combined with synthetic bone grafts (PRO-DENSE). The average age of the patients at the time of surgery was 48 years. ${ }^{8}$ The clinical failure was defined as conversion to THA or progression in head collapse. At the conclusion of the study, three hips at stage IIC and eight hips at stage IIIA were converted to THA in an average of 8.5 months postoperatively. Advanced collapse of the femoral head while awaiting THA was observed in the other six hips. Of the 19 hips, only 2 hips (10.5\%) survived without further collapse in the 5-year follow-up. This resulted in an $89.5 \%$ failure rate with early resorption of the grafting in an average of 5.3 months.

These results suggested that core decompression, combined with an injectable calcium sulfate and calcium phosphate composite graft (PRO-DENSE), was associated with high failure rates in the early postoperative period.

Recently surgeons have considered a core decompression associated with autologous bone marrow aspirates, which contain osteoprogenitor cells, and growth factors with osteoinductive (bone morphogenetic protein) or angiogenic potential (vascular endothelial growth factor) to enhance bone repair in the femoral head. ${ }^{20}$

Hernigou and Beaujean did a prospective study of 189 hips in 116 patients treated with autologous bone marrow grafting and core decompression. ${ }^{21}$ Six percent of patients with precollapsed hips required a total hip replacement (stage I and II); 57\% of patients with radiological signs of collapse before the core decompression needed an arthroplasty (stage III and IV). The success rate was higher in patients that received increased numbers of progenitor cells.

Persiani et al analyzed the clinical outcome of a series of patients affected by avascular necrosis (AVN) of the femoral head and treated with core-decompression technique and autologous stromal cells of the bone marrow. ${ }^{22}$ Twenty-nine patients with 31 hips were treated. The clinical and radiological outcome has been assessed through self-administered questionnaires (Harris Hip Score, visual analog score [VAS], and Short Form 12), X-ray, and magnetic resonance. Of all the examined hips, 25 showed relief of the symptoms and a resolution of the osteonecrosis, 11 of these were at stage I and 14 at stage II. Progression of the disease occurred in six hips ( 2 stage II, 2 stage III, and 2 stage IV).

Tabatabaee et al recently evaluated the effects of core decompression and concentrated bone marrow implantation on ONFH, recruiting 28 hips with early ONFH, randomly assigned into two groups of core decompression with (group A) and without (group B) bone marrow injection. ${ }^{23}$ The mean Western Ontario and McMaster Universities Arthritis Index (WOMAC) and VAS scores in all patients improved significantly $(p<0.001)$. MRI showed a significant improvement in group A $(p=0.046)$ and significant worsening in group B $(p<0.001)$.

In 2012, Sen et al treated 51 osteonecrotic hips in 40 patients randomizing into two treatment groups. Patients in group A (25 hips) were treated with core decompression, and those in group B (26 hips) received autologous bone marrow mononuclear cell instillation into the core tract after core decompression. ${ }^{24}$ The clinical score and hip survival were significantly better in group B than in group $A(p<0.05)$.

A review of four studies and 219 hips compared the use of core decompression alone or in association with MSCs and highlighted that MSCs lead to better results in terms of progressed vascularization and clinical results (Harris Hip score). ${ }^{25}$

A small prospective study conducted by Gangji et al compared core decompression with implantation of autogenous bone marrow cells with core decompression alone. ${ }^{26}$ Bone marrow implantation significantly reduced pain and disease progression at a 5-year follow-up. In the bone marrow group, 3 of 13 hips (23\%) progressed, compared with 8 of 11 hips (73\%) in the control core decompression group. However, the need for THA was not significantly reduced in the bone marrow group ( 2 of 13 hips [15\%]) versus the control group (3 of 11 hips [27\%]).

As regards use of PRP, in 2014 Pak et al reported a case of a 43-year-old man with early stage (stage 1) AVN of the femoral head treated with adipose tissue-derived stem cells 
and PRP. ${ }^{11}$ Patient's severe hip pain was considerably improved at 3 months after treatment, with pain scores, ROM, and MRI showing near complete resolution of necrosis. Pain scores, ROM, and MRI at 18 and 21 months after treatment indicated complete resolution of AVN.

Our hypothesis was that a new $\mathrm{CaSO}_{4} / \mathrm{CaPO}_{4}$ synthetic composite graft, the PRO-DENSE, in association with PRP, and autologous mesenchymal cells, could have several theoretical advantages in enhancing the success rate of core decompression: the PRO-DENSE provides mechanical support, MSCs and PRP induce an osteogenic activity and stimulate bone healing thanks to the platelets' growth factors contained in $\alpha$ granules such as PDGF, TGF-B, bFGF, EGF, and VEGF that play a critical role in tissue healing, and differentiation of MSCs. ${ }^{9-11}$

The cumulative survivorship at 6.3 years was $50 \%$, but this was elevated to $80 \%$ considering only patients in stage I and II.

The overall failure rate was 50\% (12 hips): two patients were in stage II (25\%), six patients were in stage III (60\%), and four patients were in stage IV (100\%).

Our results were similar to those of Rajagopal who conducted a literature review of three studies with isolated core decompression. ${ }^{27}$ Stratification based on Ficat stages demonstrates failure of core decompression in later stages. The incidence of conversion to THA with stage I and II disease was 0 to $16.7 \%$ and 17 to $43.8 \%$, respectively. However, $66 \%$ of patients with stage III needed a THA after a minimum 2-year follow-up. These results are comparable to our findings but in shorter follow-up, confirming that biological supplementation can enhance healing and delay hip replacements.

According to the literature data, best results were obtained for patients at early stage of osteonecrosis, before the collapse of the femoral head. ${ }^{1-3,7,18,27}$

A significantly higher cumulative survival rate was found for patients in early stage compared with patients in advanced stage (80\% versus $28.6 \%$ ). Although clinical outcomes improved in early and advanced ONFH stage after the core decompression with biological supplementation, patients in early stage had better results compared with patients in advanced stage.

A significant association was found between ONFH stage and the rate of failure of this procedure: patients in stage I + II had an inferior failure rate compared with stage III + IV $(p<0.05)$. The risk of failure was higher in patients at stage III and IV, as an irreversible damage of the joint is already in place. Despite the small number of patients studied and lack of control group, this study has showed a positive effect of PRP and MSCs supplementation in the treatment of ONFH.

This study has several limitations: first of all the limited number of patients; furthermore, we mixed four different techniques (core decompression, PRP, MSCs, and synthetic bone graft). In this way it is impossible to clearly know which of the procedures was truly effective in improving outcomes.

In conclusion, our results suggest that the technique is safe and good preliminary results were obtained in patients with early stages of the disease with no reported complications.

\section{References}

1 Moya-Angeler J, Gianakos AL, Villa JC, Ni A, Lane JM. Current concepts on osteonecrosis of the femoral head. World J Orthop 2015;6(08):590-601

2 Mont MA, Zywiel MG, Marker DR, McGrath MS, Delanois RE. The natural history of untreated asymptomatic osteonecrosis of the femoral head: a systematic literature review. J Bone Joint Surg Am 2010;92(12):2165-2170

3 Amanatullah DF, Strauss EJ, Di Cesare PE. Current management options for osteonecrosis of the femoral head: part 1, diagnosis and nonoperative management. Am J Orthop 2011;40(09): E186-E192

4 Ficat RP, Arlet J. Necrosis of the femoral head. In: Hungerford DS, ed. Ischemia and Necrosis of Bone. Baltimore, MD: Williams \& Wilkins; 1980:171-182

5 Issa K, Pivec R, Kapadia BH, Banerjee S, Mont MA. Osteonecrosis of the femoral head: the total hip replacement solution. Bone Joint J 2013;95-B(11, Suppl A):46-50

6 D'Ambrosi R, Marciandi L, Frediani PV, Facchini RM. Uncemented total hip arthroplasty in patients younger than 20 years. J Orthop Sci 2016;21(04):500-506

7 Zalavras CG, Lieberman JR. Osteonecrosis of the femoral head: evaluation and treatment. J Am Acad Orthop Surg 2014;22(07): 455-464

8 Yu PA, Peng KT, Huang TW, Hsu RW, Hsu WH, Lee MS. Injectable synthetic bone graft substitute combined with core decompression in the treatment of advanced osteonecrosis of the femoral head: a 5-year follow-up. Biomed J 2015;38(03):257-261

9 Rackwitz L, Eden L, Reppenhagen S, et al. Stem cell- and growth factor-based regenerative therapies for avascular necrosis of the femoral head. Stem Cell Res Ther 2012;3(01):7

10 Kuroda Y, Asada R, So K, et al. A pilot study of regenerative therapy using controlled release of recombinant human fibroblast growth factor for patients with pre-collapse osteonecrosis of the femoral head. Int Orthop 2016;40(08):1747-1754

11 Pak J, Lee JH, Jeon JH, Lee SH. Complete resolution of avascular necrosis of the human femoral head treated with adipose tissuederived stem cells and platelet-rich plasma. J Int Med Res 2014;42 (06):1353-1362

12 Meloni MC, Hoedemaeker WR, Fornasier V. Failed vascularized fibular graft in treatment of osteonecrosis of the femoral head. A histopathological analysis. Joints 2016;4(01):24-30

13 Guerado E, Caso E. The physiopathology of avascular necrosis of the femoral head: an update. Injury 2016;47(Suppl 6):S16-S26

14 Fairbank AC, Bhatia D, Jinnah RH, Hungerford DS. Long-term results of core decompression for ischaemic necrosis of the femoral head. J Bone Joint Surg Br 1995;77(01):42-49

15 Smith SW, Fehring TK, Griffin WL, Beaver WB. Core decompression of the osteonecrotic femoral head. J Bone Joint Surg Am 1995; 77(05):674-680

16 Lieberman JR, Engstrom SM, Meneghini RM, SooHoo NF. Which factors influence preservation of the osteonecrotic femoral head? Clin Orthop Relat Res 2012;470(02):525-534

17 Hong YC, Zhong HM, Lin T, Shi JB. Comparison of core decompression and conservative treatment for avascular necrosis of femoral head at early stage: a meta-analysis. Int J Clin Exp Med 2015;8(04):5207-5216

18 Castro FP Jr, Barrack RL. Core decompression and conservative treatment for avascular necrosis of the femoral head: a metaanalysis. Am J Orthop 2000;29(03):187-194

19 Bednarek A, Atras A, Gągała J, Kozak Ł. Operative technique and results of core decompression and filling with bone grafts in the treatment of osteonecrosis of femoral head. Ortop Traumatol Rehabil 2010;12(06):511-518

20 Lieberman JR, Conduah A, Urist MR. Treatment of osteonecrosis of the femoral head with core decompression and human bone morphogenetic protein. Clin Orthop Relat Res 2004;(429): 139-145 
21 Hernigou P, Beaujean F. Treatment of osteonecrosis with autologous bone marrow grafting. Clin Orthop Relat Res 2002;(405): 14-23

22 Persiani P, De Cristo C, Graci J, Noia G, Gurzì M, Villani C. Stagerelated results in treatment of hip osteonecrosis with coredecompression and autologous mesenchymal stem cells. Acta Orthop Belg 2015;81(03):406-412

23 Tabatabaee RM, Saberi S, Parvizi J, Mortazavi SM, Farzan M. Combining concentrated autologous bone marrow stem cells injection with core decompression improves outcome for patients with early-stage osteonecrosis of the femoral head: a comparative study. J Arthroplasty 2015;30(9, Suppl):11-15

24 Sen RK, Tripathy SK, Aggarwal S, Marwaha N, Sharma RR, Khandelwal N. Early results of core decompression and autologous bone marrow mononuclear cells instillation in femoral head osteonecrosis: a randomized control study. J Arthroplasty 2012;27(05):679-686

$25 \mathrm{LiX}, \mathrm{Xu}$ X, Wu W. Comparison of bone marrow mesenchymal stem cells and core decompression in treatment of osteonecrosis of the femoral head: a meta-analysis. Int J Clin Exp Pathol 2014;7(08): 5024-5030

26 Gangji V, De Maertelaer V, Hauzeur JP. Autologous bone marrow cell implantation in the treatment of non-traumatic osteonecrosis of the femoral head: five year follow-up of a prospective controlled study. Bone 2011;49(05):1005-1009

27 Rajagopal M, Balch Samora J, Ellis TJ. Efficacy of core decompression as treatment for osteonecrosis of the hip: a systematic review. Hip Int 2012;22(05):489-493 